\title{
Contracture Development in Whales
}

\author{
Jessica Pingel ${ }^{1 *}$, Adrian Harrison ${ }^{2}$ \\ ${ }^{1}$ Department of Neuroscience, Faculty of Health \& Medical Sciences, University of Copenhagen, Frederiksberg C, Denmark \\ ${ }^{2}$ PAS, Physiology, Faculty of Health \& Medical Sciences, University of Copenhagen, Frederiksberg C, Denmark \\ Email: *jpingel@sund.ku.dk
}

How to cite this paper: Pingel, J. and Harrison, A. (2020) Contracture Development in Whales. Open Journal of Marine Science, 10, 173-176.

https://doi.org/10.4236/ojms.2020.103013

Received: June 4, 2020

Accepted: July 12, 2020

Published: July 15, 2020

Copyright $\odot 2020$ by author(s) and Scientific Research Publishing Inc. This work is licensed under the Creative Commons Attribution International License (CC BY 4.0).

http://creativecommons.org/licenses/by/4.0/

\begin{abstract}
It is well known that whales in captivity suffer from dorsal fin bending. However, the mechanisms behind this change are poorly understood. Humans can suffer from similar symptoms either after a period of immobilization or after suffering a brain lesion. Therefore, we here speculate whether the bent dorsal fins are reflecting contracture development in whales.
\end{abstract}

\section{Keywords \\ Dorsal Fin, Cetaceans, Contracture, Immobilization, CNS Lesion}

\section{Introduction/Discussion}

Bent dorsal fins are a phenomenon that has primarily been documented in whales in captivity, but a recent study has shown that fin bending also occurs in free-living cetaceans [1]. Previous reports on cetaceans in captivity showed that the dorsal fins of these whales were completely collapsed and thus lost their function entirely (see example Figure 1(a)). Nevertheless, the reason for this abnormality remains unknown.

In humans, stiff and bent limbs that are stuck in awkward positions are called contractures, and are common complications after lesions to the central nervous system (CNS) [2]. This includes cerebral palsy (CP), stroke survivors and traumatic brain injury patients. However, contractures can also be caused by immobilization especially when a muscle is immobilized in a shortened position [3]. Common pathologies causing immobilization related contractures are Rheumatoid arthritis, prolonged bed-rest and bone fractures [4]. In humans, contractures can develop in both upper- and lower extremities. The severity of contractures can vary and in the worst cases the joints lose their function entirely. Figure 1(b) shows an example of a wrist with a very severe contracture. This individual has $\mathrm{CP}$ and is actually born with two normal functioning hands, but due to contrac- 
ture development his wrist has now lost its function and can no longer move, although it is the source of chronic pain.

The reversibility of contractures depends on the severity, and since contracture development worsens over time early interventions towards contracture development are crucial [5]. When contractures develop in neurological disorders, an altered neural drive to the muscles is a logical suspect, therefore it has been generally assumed that spasticity is the main cause of contractures [6]. However, there is a growing body of evidence indicating that contractures develop also when spasticity is absent. A 10-year follow-up study showed that recipients of a dorsal rhizotomy still developed contractures [7]. A rhizotomy is the most radical treatment against spasticity that eliminates spasticity by cutting the dorsal roots in the spinal cord. A new growing perception is that contracture development is caused by multiple factors, and that collagen accumulation and stiffness changes in the extracellular matrix are among the key explanations for contracture development in neurological disorders [8].

Immobilization provoked contractures result in similar adaptations causing an accumulation of collagen and increased passive stiffness of the joint [3]. Interestingly, those contractures that are induced by immobilization are reversible with regard to the population of sarcomeres [9], and the range of motion [5] when treated in time. Contractures that are caused by CNS lesions are not reversible and the joints of these patients are affected life-long [10]. We therefore raise the hypothesis that whales in captivity exhibiting bent dorsal fins, may be suffering from immobilization-related contractures. It is generally assumed that whales like the Orcinus orca swim at least 100 miles a day and can dive to depths of hundreds of feet. Creating an environment in captivity where these criteria can be met is unthinkable and therefore all the present captive quarters should be considered as restrictive with regard to the normal physical activity of whales, and therefore likely to induce different degrees of immobilization. Thus, housing

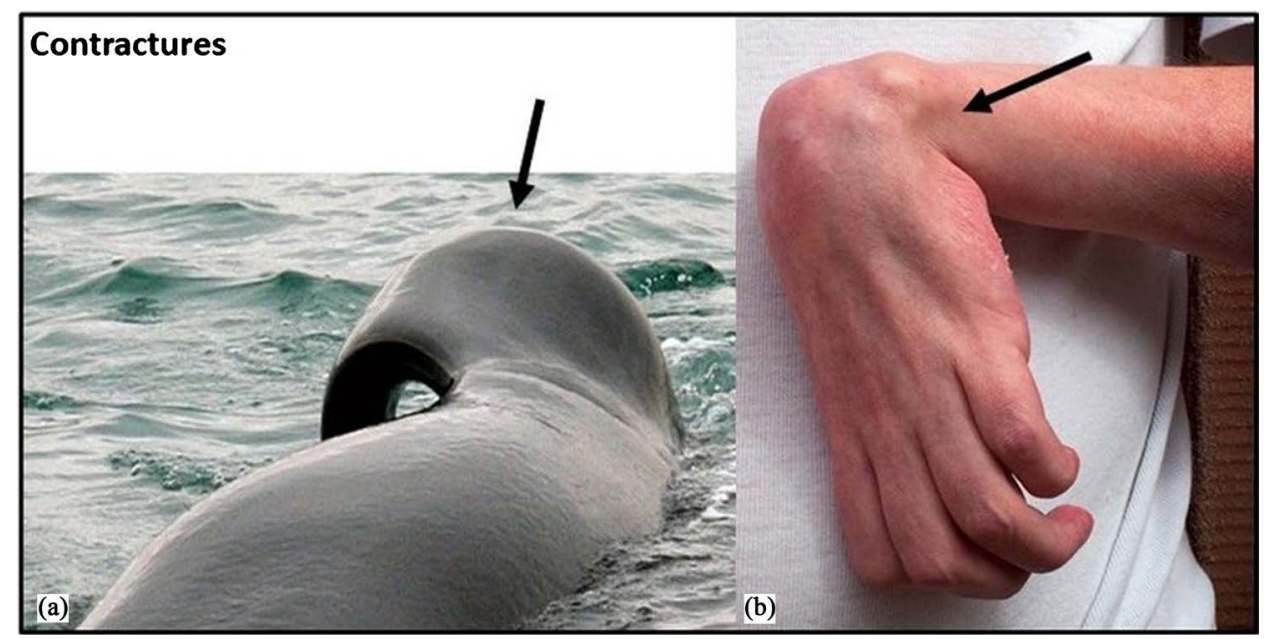

Figure 1. Photographs of contractures: in (a) the dorsal fin of a killer whale (Orcinius orca), and (b) the left wrist of a human subject with a brain lesion (Cerebral Palsy). The black arrows indicate the site of contracture. 
cetaceans in restrictive quarters may well in itself lead to immobilization-related fin contractures through restricted natural swimming behavior. Whales in captivity are not born with bent dorsal fins, but they develop them over time, in exactly the same way as disease progression occurs for CP patients. It is for this reason that we want to emphasize the need for early interventions towards prevention of contracture development for newborn captive whales as being crucial in order to prevent dorsal fin bending. Especially because it is still unclear how fin bending affects the movement abilities of the whales and whether it causes the animals pain as it does in human patients with contractures.

A second, additional hypothesis that we want to raise is that free-living whales with bent dorsal fins may suffer from CNS lesion-related contractures. The brain is a very delicate organ in all species and all animals can suffer hypoxia induced brain lesions and even neurological disorders such as stroke. For instance, even cetaceans can drown if they are hindered in some way from reaching the water's surface to breath. Therefore, it is not inconceivable that free-living whales may suffer a minor brain injury through a blow to the head or back, by a ship's propeller or by similar heavy equipment. Entanglement in fishing nets may likewise result in hypoxia induced CNS lesions.

The possibility of contracture development in whales has until now been overlooked and the cause for bent fins has rather been attributed to less likely causes such as loss of collagen in cetacean fins with increasing age, or as a result of long-term exposure of fins to the warm air associated with their restrictive housing. Thus, there is now an acute need for innovation in this area. It is time to re-think the cause of abnormal fin bending in whales. Fin bending in both free and captive whales still has many unresolved issues including whether the fin bending solely restricts their movement, or additionally causes them pain during movement. A better understanding of why and how the dorsal fins are bending might help in the implementation of preventative procedures for both whales in captivity and free-ranging whales. It is especially important that such interventions be sought for newborn whales in captivity to avoid the development of severe contractures. Finally, even though the application of preventive interventions on free living animals is unrealistic, a better understanding of the mechanisms leading to fin bending is a necessary first step that might help us to create tools that can help these animals in the near future.

\section{Conclusion}

In summary, we suggest that dorsal fin bending in whales kept in captivity is the result of contracture development due to immobilization. In addition, we propose that bent dorsal fins in free-living whales arise through contracture development after acquiring a CNS lesion.

\section{Acknowledgements}

We thank Dr. Peter C. Raffalt from the Norwegian School of Sport Sciences for his inspiration, good scientific discussions and his helpful critical reading. 


\section{Conflicts of Interest}

The authors declare no conflicts of interest regarding the publication of this paper.

\section{References}

[1] Alves, F., et al. (2018) The Incidence of Bent Dorsal Fins in Free-Ranging Cetaceans. The Journal of Anatomy, 232, 263-269. https://doi.org/10.1111/joa.12729

[2] O’Dwyer, N.J., Ada, L. and Neilson, P.D. (1996) Spasticity and Muscle Contracture Following Stroke. Brain, 119, 1737-1749. https://doi.org/10.1093/brain/119.5.1737

[3] Baker, J.H. and Matsumoto, D.E. (1988) Adaptation of Skeletal Muscle to Immobilization in a Shortened Position. Muscle \& Nerve, 11, 231-244. https://doi.org/10.1002/mus.880110308

[4] Born, C.T., Gil, J.A. and Goodman, A.D. (2017) Joint Contractures Resulting from Prolonged Immobilization: Etiology, Prevention, and Management. Journal of the American Academy of Orthopaedic Surgeons, 25, 110-116. https://doi.org/10.5435/JAAOS-D-15-00697

[5] Trudel, G. and Laneuville, O. (2014) Quantitative and Temporal Differential Recovery of Articular and Muscular Limitations of Knee Joint Contractures; Results in a Rat Model. The Journal of Applied Physiology, 117, 730-737. https://doi.org/10.1152/japplphysiol.00409.2014

[6] Hagglund, G. and Wagner, P. (2011) Spasticity of the Gastrosoleus Muscle is Related to the Development of Reduced Passive Dorsiflexion of the Ankle in Children with Cerebral Palsy: A Registry Analysis of 2796 Examinations in 355 Children. Acta Orthopaedica, 82, 744-748. https://doi.org/10.3109/17453674.2011.618917

[7] Tedroff, K., et al. (2011) Does Loss of Spasticity Matter? A 10-Year Follow-Up after Selective Dorsal Rhizotomy in Cerebral Palsy. Developmental Medicine \& Child Neurology, 53, 724-729. https://doi.org/10.1111/j.1469-8749.2011.03969.x

[8] Pingel, J., Bartels, E.M. and Nielsen, J.B. (2016) New Perspectives on the Development of Muscle Contractures Following Central Motor Lesions. The Journal of Physiology, 595, 1027-1038. https://doi.org/10.1113/JP272767

[9] Tabary, J.C., et al. (1972) Physiological and Structural Changes in the Cat's Soleus Muscle Due to Immobilization at Different Lengths by Plaster Casts. The Journal of Physiology, 224, 231-244. https://doi.org/10.1113/jphysiol.1972.sp009891

[10] Colver, A., Fairhurst, C. and Pharoah, P.O. (2014) Cerebral Palsy. Lancet, 383, 1240-1249. https://doi.org/10.1016/S0140-6736(13)61835-8 